\title{
Qualité des eaux du Golfe du Morbihan - Utilisation d'urn modèle hydrodynamique
}

\author{
Jean-Marc Janin, Frédéric Marcos \\ Ingénieurs-chercheurs au Laboratoire National d'Hydraulique, EDF, Chatou
}

\begin{abstract}
Considering the complexity of the morphology of Morbihan Gulf, the numerical model proved to be an essential tool to study water quality in this area. This is all the more obvious since the goals to reach are not only to be able to reproduce present time sanitary conditions of the Gulf, a sort of validation-understanding phase, but also to assist decision makers for future developments. The first model we built is a $2 D$ model, calibrated for time-scales between one hour and one week. It proved that it was particularly well adapted to model pollutants with short lifetime like faecal bacteria, one of main pollutant source around the Gulf. For nitrogeneous constituents, other principal source of pollution there, a model that covers larger time-scales is necessary, that should be the logical result of this work.
\end{abstract}

\section{Introduction}

Le Golfe du Morbihan, situé en Bretagne Sud, est un système maritime très complexe. Sa superficie, de l'ordre de $125 \mathrm{~km}^{2}$, est très importante comparée à la taille de sa liaison avec la baie de Quiberon, dont la largeur est inférieure à $1 \mathrm{~km}$ (fig.1). On y trouve de nombreuses îles dont les 2 principales, situées dans sa partie centrale, sont l'Mle aux Moines et l'Ile d'Arz. La situation et le climat du site ont permis son développement touristique et la qualité des eaux est désormais un enjeu majeur pour les collectivités locales. Dans le cadre d'un pré-contrat de baie, le Conseil Général du Morbihan a donc confié au LNH la réalisation d'un modèle numérique destiné à étudier le devenir des polluants rejetés dans le Golfe et ses abords immédiats.

L'intérêt de l'outil de modélisation est non seulement de décrire finement l'état sanitaire actuel de ce domaine complexe mais aussi et surtout de servir d'aide à la décision en étudiant l'impact sur le milieu de tel ou tel projet d'aménagement. Dans cet article nous ne présentons que la première phase, c'est à dire la construction et la validation du modèle dans la situation actuelle. Un premier cas d'application a d'ores et déjà été réalisé, il concerne l'optimisation du rejet de la station d'épuration d'Arzon. 
Le choix des éléments finis s'est imposé naturellement, il permet en effet de disposer d'un maillage offrant toute la souplesse pour décrire les nombreuses singularités (point de rejets, détroits, forts gradients de fond,...) et représenter précisément le trait de côte, très tourmenté dans la zone d'étude (fig.1).

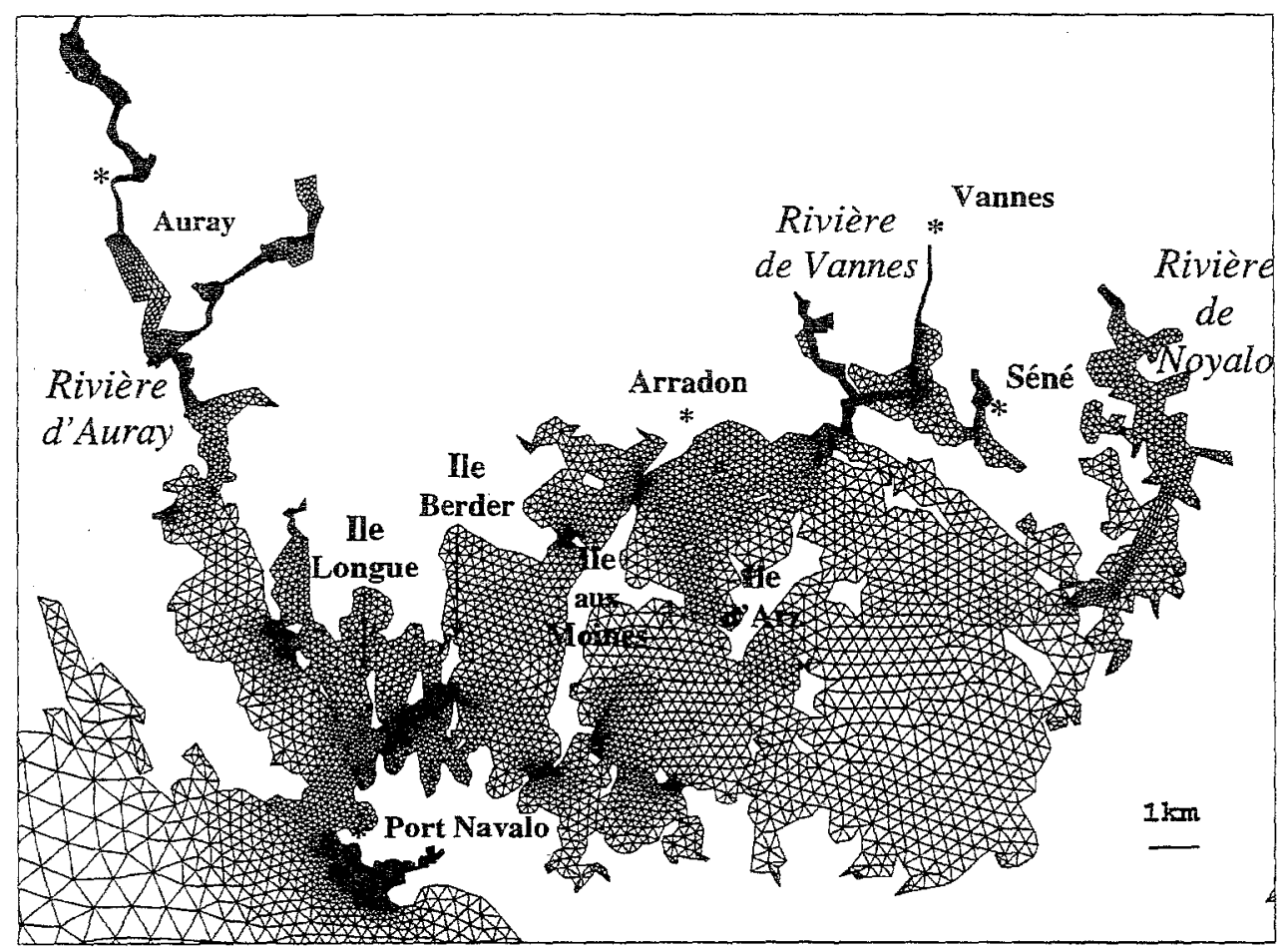

Figure 1 : Maillage et points de repère

L'emprise du modèle déborde très largement le Golfe puisque celui-ci s'étend jusqu'à une ligne reliant Le Croisic à Quiberon en passant par Belle-Ile. Les conditions limites sur cette ligne ont été obtenues à partir d'un modèle du LNH de 30000 éléments des Côtes Ouest de France [3]. A l'intérieur du Golfe pas moins de 34 îles ont été représentées. Le maillage comporte près de 16000 éléments dont la taille varie d'environ $1 \mathrm{~km}$ dans le Mors Bras à $200 \mathrm{~m}$ en moyenne dans le Golfe, voir $50 \mathrm{~m}$ dans les détroits. La construction de ce maillage a pris en compte au mieux la bathymétrie complexe de la zone, on note en particulier la présence de nombreux bancs découvrants et d'une fosse au niveau de Port-Navalo.

Les polluants que nous modélisons sont considérés comme passifs vis à vis de l'hydrodynamique. Cette hypothèse, justifiée par la nature de ceux-ci, est importante car elle permet de dissocier dans notre méthodologie le calcul dynamique de la marée et de ses courants, de l'action de ceux-ci sur les paramètres de qualité d'eau. Nous aborderons donc tour à tour ces 2 aspects.

\section{Hydrodynamique}

La part essentielle des mouvements de masses d'eau dans ce secteur est due à l'action de la marée, dont voici quelques caractéristiques. 


\subsection{Doñuées maturelles}

\subsubsection{Niveaux}

A l'entrée du Golfe à Port-Navalo, le mamage est respectivement de $4,6 \mathrm{~m}$ et $2,2 \mathrm{~m}$ en Vive Eau et en Morte Eau. L'intérieur du Golfe se divise ensuite grossièrement en 3 zones. Une première zone à l'Ouest de l'Ile de Longue, comprend la rivière d'Auray, la marée y possède environ les mêmes caractéristiques (amplitude, phase) qu'à Port-Navalo. Une seconde zone s'étend entre l'Ile Longue et l'Ile Berder, le marnage est de l'ordre de 3,2 $\mathrm{m}$ en Vive Eau et le déphasage d'une heure avec PortNavalo. Une troisième zone se situe à l'Est de l'Ile Berder, le marnage est d'environ $2,8 \mathrm{~m}$ en Vive Eau et le déphasage avec Port-Navalo varie de $1 \mathrm{~h} 40$ à $2 \mathrm{~h} 30$ d'Ouest en Est.

\subsubsection{Courants}

Les volumes d'eau entrant et sortant du Golfe du Morbihan au cours d'un cycle de marée sont très importants et impliquent l'existence de courants violents dans les détroits. Les courants les plus importants ont été mesurés entre l'Mle Berder et l'Ile de la Jument, soit plus de 9 noeuds en marée de Vive Eau exceptionnelle. La direction des courants, d'une manière générale, est fortement conditionnée par la morphologie du site où apparaissent très nettement de nombreux chenaux.

\subsection{Modèle}

\subsubsection{Présentation}

Le modèle utilisé pour reproduire l'hydrodynamique complexe de la zone s'appuie sur le logiciel TELEMAC-2D [4] qui résout les équations de Saint-Venant et suppose l'homogénéité des courants sur la verticale. Cette hypothèse est valide en mer à marée en l'absence de stratification, c'est à dire ici dans la quasi totalité de la zone.

Les paramètres physiques pris en compte pour le forçage du modèle sont l'onde de marée à la limite au large et les débits des différentes sources et rivières. Des cas de calcul prenant en compte les effets d'un vent uniforme ont également été réalisés. En revanche, compte tenu de la relativement faible emprise du modèle, ni la force de Coriolis, ni l'attraction des masses d'eau à l'intérieur même du modèle par les astres n'ont été considérées. On aura eu soin de vérifier au préalable le bien fondé de ces approximations.

\subsubsection{Validation}

Le modèle s'est attaché dans un premier temps à reproduire les données naturelles. Nous nous sommes plus particulièrement intéressés aux caractéristiques sur les niveaux car ce sont ces paramètres qui pilotent les échanges entre les différentes zones du Golfe et l'extérieur, les courants n'étant en fait que la résultante de ces mouvements de surface.

Nous disposons de données du SHOM en Vive Eau et Morte Eau pour 7 points bien répartis. Nous présentons page suivante une comparaison sur 2 d'entre eux : 


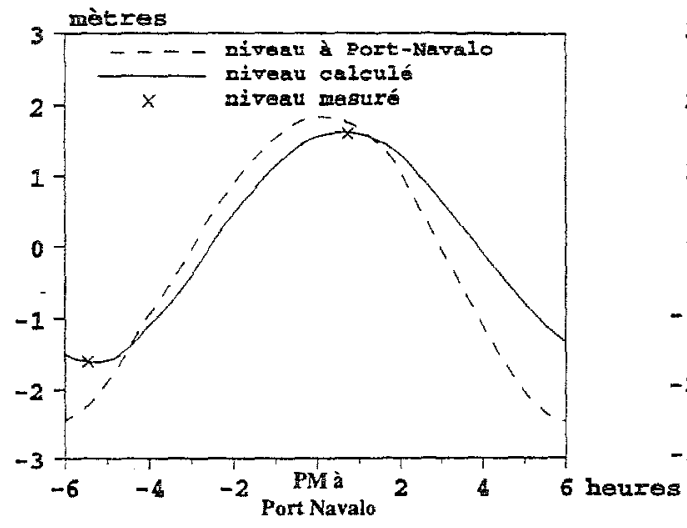

Ile Gavrinis (entre l'He Longue et Berder)

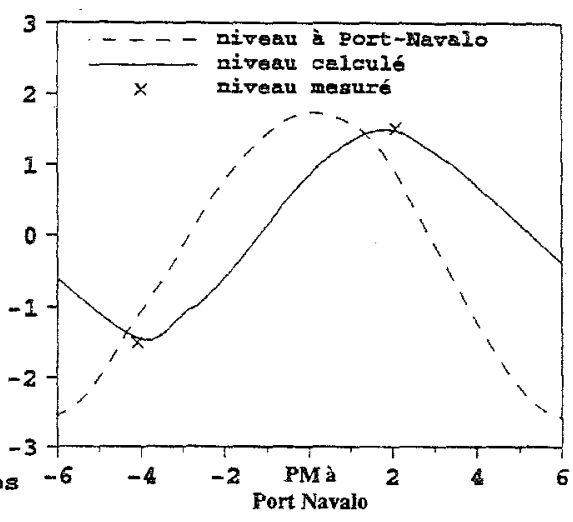

Vannes

Figure 2 : Comparaison mesures-modèle et déphasage par rapport à Port-Navalo On note un très bon comportement du modèle, en particulier sur les marnages qui ne s'écartent jamais de plus de $10 \mathrm{~cm}$ par rapport à la mesure. Concernant les phases, on note de très bons résultats sur l'instant de la Pleine Mer qui s'écarte au maximum de 13 minutes entre mesures et calcul. C'est en revanche un peu plus médiocre à Basse Mer avec 2 écarts supérieurs à 25 minutes, Auray en Vive Eau et Vannes en Morte Eau. Ces retards s'expliquent par une surestimation des lits des rivières à Basse Mer par le modèle et donc une amplification des mécanismes inertiels : les lits des rivières à Basse Mer sont en effet, dans bon nombre d'endroits en nature, particulièrement étroits et nécessiteraient un maillage encore plus fin pour être correctement représentés.

\subsubsection{Apports du modèle}

Dans le domaine de l'hydrodynamique, bien qu'il s'agisse du domaine le mieux décrit, le modèle a d'ores et déjà apporté des éléments nouveaux et ce essentiellement dans 2 registres qui intéressent la qualité d'eau : les volumes oscillants et les temps de résidence. Ces registres, qui correspondent à des notions très intégrées de l'hydrodynamique, sont effectivement délicats à évaluer sur le terrain.

\subsubsection{Volumes oscillants}

Les volumes oscillants, que nous présentons figure 3, correspondent à la moyenne des flux en flot et en jusant échangés entre zones pendant le cycle de marée de $12 \mathrm{~h} 25$. Chacun de ces flux a été obtenu en intégrant, sur la période considérée, le débit instantanné calculé toutes les 5 secondes. Compte tenu de la périodicité du signal de marée, ces volumes en première approximation sont identiques entre le flot, période de remplissage, et le jusant, période de vidange, ce qui justifie notre moyenne.

En réalité, ils ne le sont pas tout à fait et ce pour 2 raisons. La première raison est que, même si le signal était effectivement rigoureusement périodique de période $12 \mathrm{~h} 25$, il existe, du fait de la présence des 2 grandes îles centrales, une circulation résiduelle apériodique autour de celles-ci. Lors d'une précédente étude [1], les volumes associés avaient été évalués à quelques millions de $\mathrm{m}^{3}$ par marée. La seconde explication, dont l'incidence se fait cette fois sentir sur la totalité du 
domaine, est que le signal de marée n'est justement pas périodique de période $12 \mathrm{~h} 25$ mais qu'il existe en particulier une période égale au demi-cycle lunaire pendant laquelle la marée passe, dans un sens puis dans l'autre, par les 3 phases étudiées (Vive Eau, marée moyenne, Morte Eau). Ainsi, en une semaine, soient 13 à 14 cycles de marée, les volumes échangés entre le Golfe et l'extérieur fluctuent de 170 à 300 millions de $\mathrm{m}^{3}$, soit un écart moyen de 10 millions de $\mathrm{m}^{3}$ d'une marée à l'autre et donc, en moyenne, une différence de 5 millions de $\mathrm{m}^{3}$ entre une phase de flot et une phase de jusant consécutive.

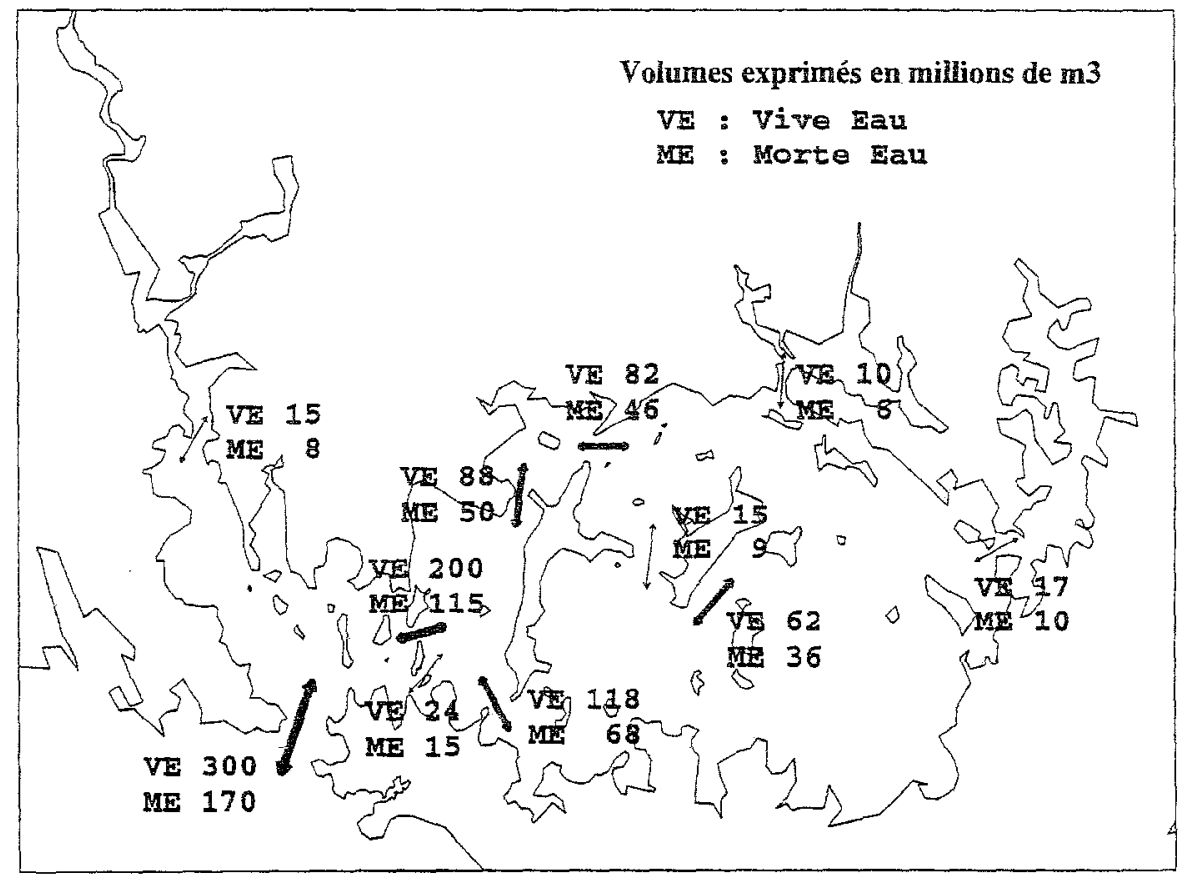

Figure 3 : Volumes échangés entre diverses parties du golfe du Morbihan

Sur la figure 3 , on note que $2 / 3$ des échanges à Port-Navalo proviennent du remplissage puis de la vidange de la partie centrale du Golfe (échanges au niveau du détroit Berder-Jument). On note également que les mouvements de masses d'eau entre les parties occidentales et orientales du Golfe s'équilibrent entre le Sud et le Nord de l'Ile aux Moines, avec respectivement 118 et 82 millions de $\mathrm{m}^{3}$ échangés. Les rivières en revanche, à cause de leur moindre surface, contribuent de manière beaucoup plus limitée aux échanges (environ 10 millions de $\mathrm{m}^{3}$ chacune).

Pour évaluer le pouvoir de renouvellement potentiel des eaux du Golfe, il convient de rapporter les volumes oscillants à un volume de référence, celui à Pleine Mer par exemple :

\begin{tabular}{|l|c|c|c|}
\hline$\left(\right.$ en millions de $\left.\mathrm{m}^{3}\right)$ & Vive Eau & Marée moyenne & Morte Eau \\
\hline Volume oscillant & 300 & 240 & 170 \\
\hline Volume à PM & 680 & 630 & 580 \\
\hline Volume oscillant & $44 \%$ & $38 \%$ & $29 \%$ \\
\hline Volume à PM & & \\
\hline
\end{tabular}

Tableau 1 : Echanges entre le Golfe et l'extérieur 
Ainsi, dans l'hypothèse d'un polluant homogènement distribué dans le Golfe à Pleine Mer, respectivement en Vive Eau et en Morte Eau, $44 \%$ et $29 \%$ de celui-ci aura pu, au mieux, être évacué d'ici à la Pleine Mer suivante. Il s'agit bien entendu de majorants car une part importante des masses d'eau évacuées au jusant rentre de nouveau au flot suivant. Pour avoir une idée plus précise de ces paramètres de renouvellement, une étude spécifique sur les temps de résidence a été faite.

\subsubsection{Temps de résidence}

Dans la figure 4, page suivante, les masses d'eau qui ont définitivement quitté le Golfe après 5 marées de Vive Eau, cas le plus favorable, sont indiquées par une tache noire placée à leur position d'origine. Il s'agit néanmoins d'interpréter ce résultat avec prudence car chaque masse d'eau considérée, positionnée initialement à un noeud du maillage, a été traitée comme une masse ponctuelle ne se mélangeant pas avec sa voisine. Qui plus est, les 5 marées prises en compte sont 5 marées identiques, ce qui ne correspond pas à la réalité, comme nous l'avons évoqué au précédent paragraphe.

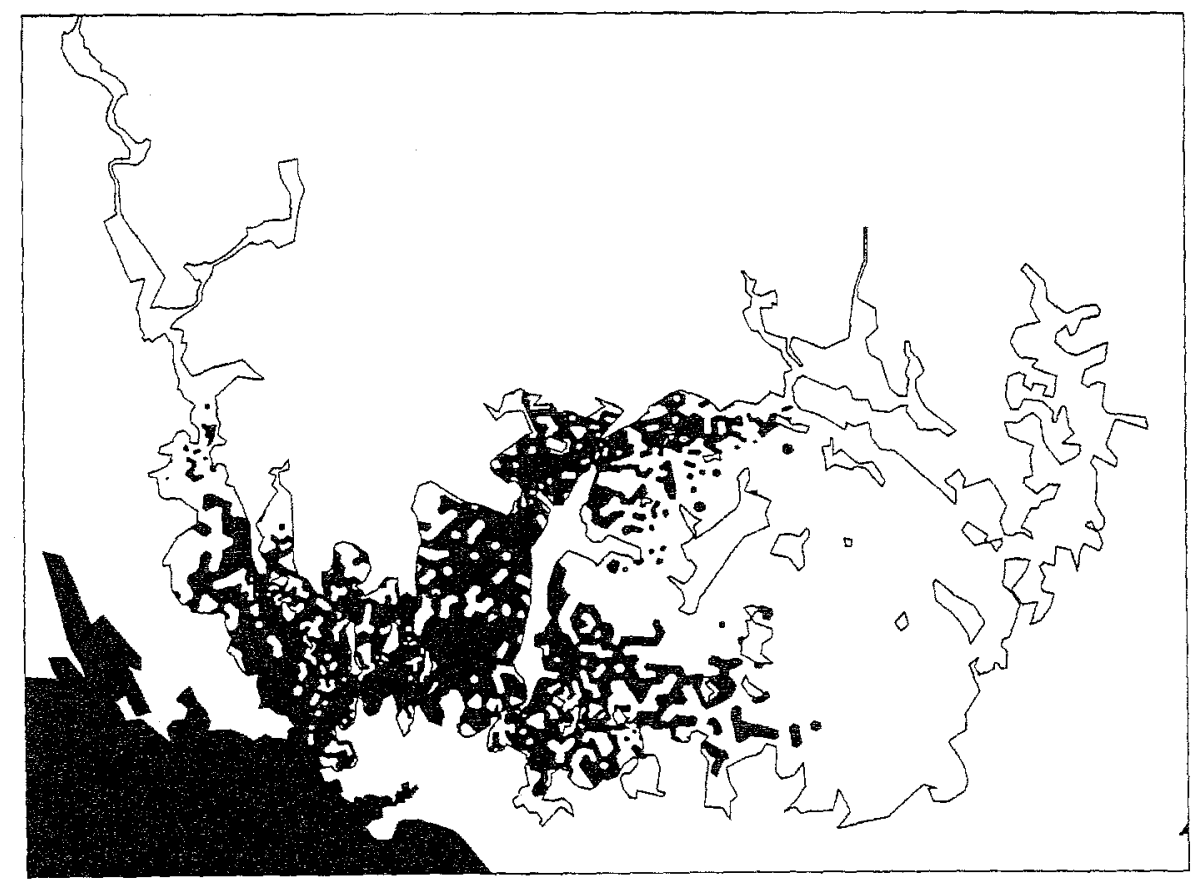

Figure 4 : Masses d'eau renouvelées après 5 marées de Vive Eau

Ce résultat permet toutefois de définir dans les grandes lignes les zones renouvelées après un délai de 2 à 3 jours. On constate en particulier que seule une zone en arc de cercle autour de l'Ile aux Moines (du Nord au Sud de l'île en passant par l'Ouest), ainsi que la partie très avale de la rivière d'Auray, sont fortement renouvelées sur cette période.

Sachant que les sources de pollution sont pour l'essentiel situées très en amont des rivières, il y a lieu de s'inquiéter sur l'échelle des temps à considérer pour atteindre un état d'équilibre. C'est le cas en particulier pour les composés azotés que l'on peut en première approximation considérer comme conservatifs dans le milieu en 
situation hivernale. Pour l'autre source principale de pollution, les coliformes, il existe un autre facteur déterminant, leur durée de vie, dont l'échelle de temps caractéristique, définie par un coefficient appelé T90, est de l'ordre de la journée. Cette échelle étant compatible avec le domaine de validité du modèle, nous nous sommes donc tournés dans un premier temps vers cette source de pollution.

\section{Pollution microbienne}

\subsection{Domnées}

En concertation étroite avec la DDE du Morbihan et la station IFREMER La Trinité, nous avons retenu 18 sources principales avec les flux journaliers en coliformes suivants, correspondant à une situation hivernale :

$$
\text { - S.E. : station d'épuration - E.P. : collecteur d'eau pluviale }
$$

\begin{tabular}{|l|c|l|l|l|l|}
\hline S.E. Le Crac'h & $110^{9}$ & S.E. Séné Cadouarn & $210^{13}$ & Rivière Le Vincin & $110^{11}$ \\
\hline S.E. Auray & $410^{10}$ & S.E. Sarzeau Le Logeo & $110^{12}$ & Rivière Le Bernus & $110^{11}$ \\
\hline S.E. Larmor Baden & $210^{9}$ & Rivière Le Loch & $110^{12}$ & E.P. Auray St Goustan & $210^{12}$ \\
\hline S.E. Ile aux Moines & $210^{12}$ & Rivière Le Sal & $210^{12}$ & E.P. Le port du Bono & $510^{10}$ \\
\hline S.E. Arradon & $610^{12}$ & Rivière La Marle & $110^{13}$ & E.P. Baden Port Blanc & $110^{11}$ \\
\hline S.E. Vannes Kermain & $610^{13}$ & Rivière Le Liziec & $710^{13}$ & E.P. Berder & $510^{10}$ \\
\hline
\end{tabular}

Tableau 2 : Principales sources microbiennes prises en compte

Nous ne présentons que la situation hivernale car c'est la saison la plus critique vis à vis de la pollution microbienne. En effet, en cette saison, les bactéries résistent mieux au milieu marin hostile et le T90 est environ doublé par rapport à l'été. Pour se convaincre du caractère déterminant de ce paramètre, nous présentons en 2 points du domaine, sur la figure 5, les concentrations calculées pour 3 valeurs distinctes du T90.

On note, comme on l'avait préssenti en étudiant les temps de résidence, que pour un T90 infini, polluant conservatif, l'équilibre n'est pas atteint après 5 jours.

\subsection{Modèle}

\subsubsection{Présentation}

Pour ce qui est du transport, de la dilution et de la disparition des coliformes le modèle utilisé s'appuie sur SUBIEF [5] qui réalise ces opérations à partir d'un champ de courant $2 \mathrm{D}$ instationnaire.

Pour le transport, la méthode des caractéristiques, qui a de bonne propriété physiques a été retenue. Pour la dilution, nous avons finalement opté pour un coefficient de $0,5 \mathrm{~m}^{2} / \mathrm{s}$ ce qui a un effet relativement limité sur la solution compte tenu de l'importance des 2 autres processus. Pour le taux de mortalité enfin, celle-ci a fait l'objet d'une étude paramétrique dont nous présentons, paragraphe suivant, quelques éléments. 


\subsubsection{Validation}

La validation du modèle, qui en l'occurence consistait à caler le T90, s'est faite à partir de concentrations mesurées par IFREMER sur des huîtres prélevées en différents points. Estimant par ailleurs que le taux de coliformes présents dans le coquillage est environ 30 fois plus important que celui dans le milieu ambiant, on déduit-la concentration dans le milieu. Un T90 de $24 \mathrm{~h}$ a permis d'obtenir un très bon ajustement du modèle avec les mesures :
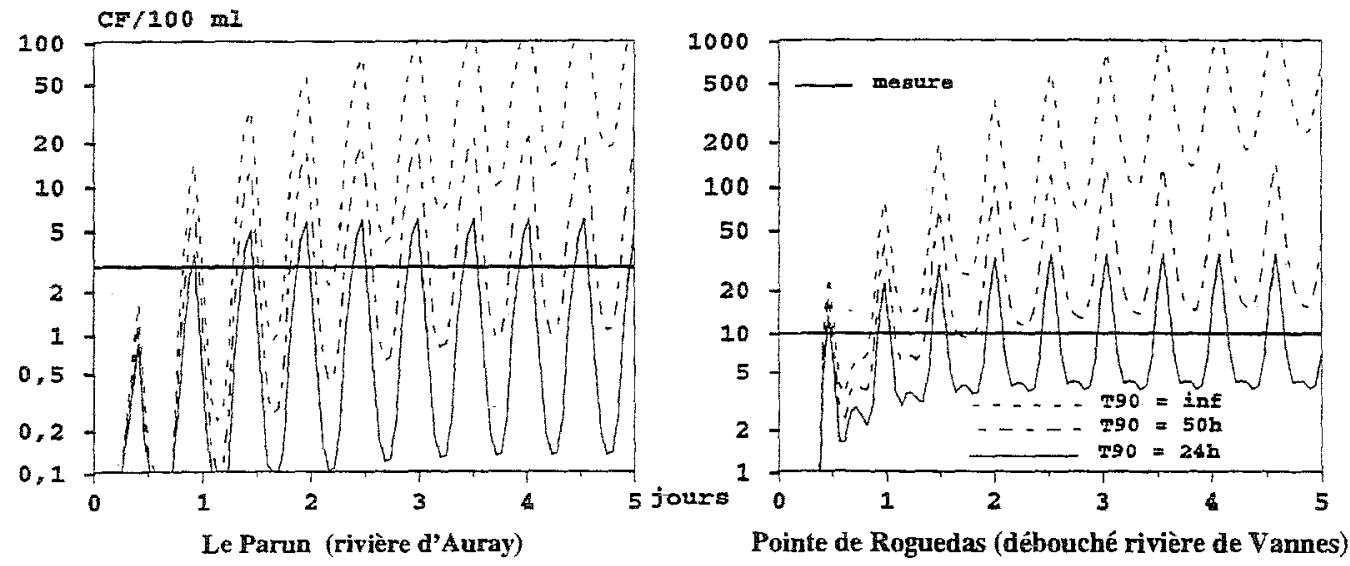

Figure 5 : Coliformes mesurés et calculés pour différents $T 90$

Cette valeur de $24 \mathrm{~h}$, d'après Pommepuy et al. [2] est tout à fait conforme à une situation d'hiver. Elle correspond à une MES de l'ordre de $10 \mathrm{mg} / \mathrm{l}$.

\subsubsection{Résultats}

Nous présentons ci-dessous les résultats les plus critiques obtenus à Basse Mer de Vive Eau, c'est à dire la situation pour laquelle les contaminants ont pu être transportés le plus en aval de leur source.

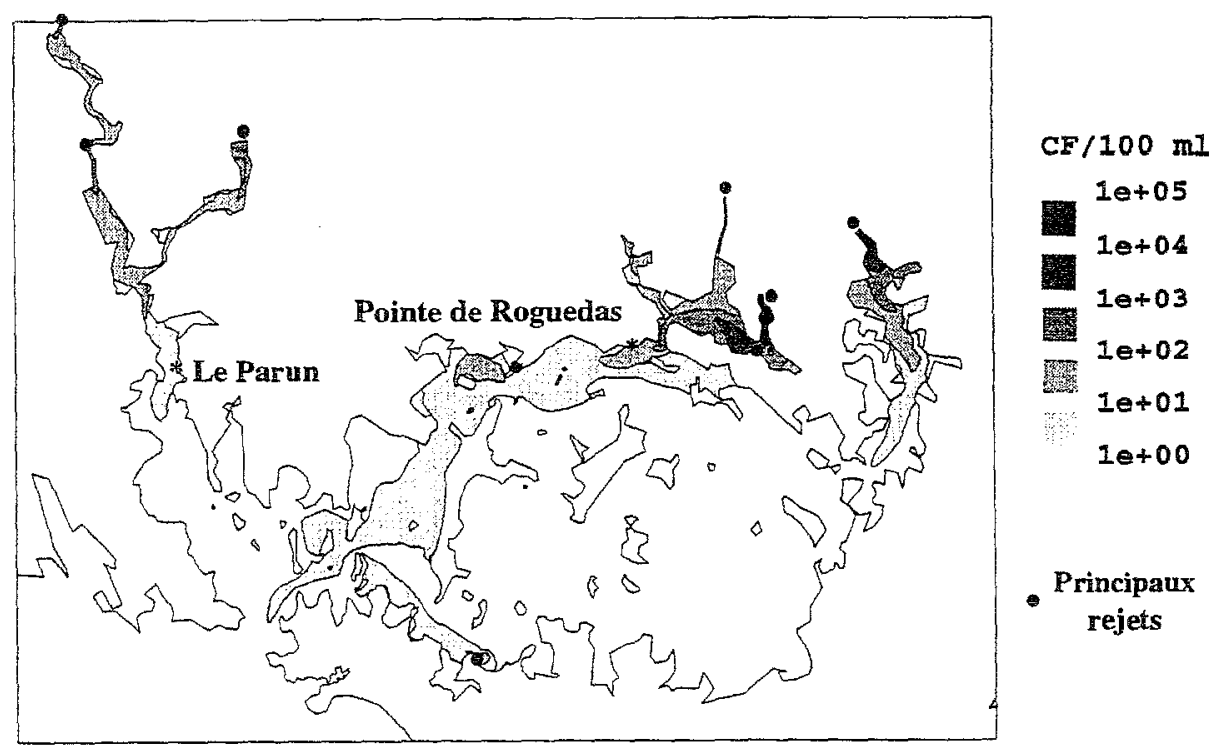

Figure 6: Concentration en coliformes à Basse Mer de Vive Eau 
Ce résultat montre que la situation est loin d'être alarmante pour le Golfe en ce qui concerne la contamination bactérienne. Une concentration inférieure à $10 \mathrm{CF} / 100$ ml, correspondant à une eau d'excellente qualité, est obtenue dans la quasi totalité du Golfe et dans les parties avales des rivières. L'eau est cependant de qualité moyenne en amont du confluent Auray-Bono, voire très médiocre dans la branche vers Séné de la rivière de Vannes ou en amont de la rivière de Noyalo. La rénovation prévue des stations d'épuration dans cette zone devrait grandement améliorer l'état sanitaire.

\section{Conclusion}

Le modèle LNH a permis, à l'échelle de quelques marées, d'apprécier finement la circulation complexe des masses d'eau dans le Golfe du Morbihan. Cette circulation, combinée à un T90 de l'ordre de $24 \mathrm{~h}$, est déterminante pour la dilution de la pollution microbienne que le modèle a correctement reproduit dans le contexte actuel. Vis à vis de cette pollution, il est donc opérationnel pour tester de nouveaux aménagements. Le cas de l'azote est plus délicat et nécessite de s'intéresser à des échelles de temps plus longues, cela devrait être la prochaine étape.

\section{Références princinales}

[1] MANAUD F. : Etude préliminaire physique et biologique du Golfe du Morbihan - CNEXO-COB Unité Littoral (1976)

[2] POMMEPUY M. et al. : Le devenir des bactéries en zone littorale - La mer et les rejets urbains - IFREMER - actes de colloques $n^{\circ} 11$ (1990)

[3] JANIN J.M., GALLAND J.C. : Numerical modelling of tidal currents along the West European continental shelf in order to predict the movements of polluted matters - Ind International Conference on Hydraulic and Environmental Modelling of Coastal, Estuarine and River Waters Bradford, England (1992)

[4] HERVOUET J.M. : Element by element methods for solving shallow water equations with F.E.M. - IXth International Conference on Computational Methods in Water Resources - Denver, U.S.A. (1992)

[5] MOULIN C., HERVOUET. J.M., JANIN J.M. : Advection/diffusion equations using finite element methods in the TELEMAC system IXth International Conference on Finite Elements in Fluids - Venezia, Italy (1995) 